\title{
ALIMENTAÇÃO FORA DE CASA E EXCESSO DE PESO: UMA ANÁLISE DOS MECANISMOS EXPLICATIVOS
}

\author{
Eating away from home and excess weight: an analysis of \\ explanatory mechanisms
}
Alimentación en la calle y el exceso de peso: un análisis de los mecanismos explicativos

Artigo de Revisão

\section{RESUMO}

Objetivo: Discutir os possíveis mecanismos da relação entre consumo de alimentos fora do domicílio e ganho excessivo de peso. Métodos: Realizou-se um levantamento bibliográfico de artigos científicos que descrevessem as características dos alimentos consumidos fora do domicílio e de artigos que investigassem a relação entre essas características e o ganho excessivo de peso, publicados nas bases de dados: MEDLINE, Biblioteca Latino-Americana e do Caribe (LILACS) e Scientific Eletronic Library Online (SciELO). Resultados: Constatouse que diversas características da alimentação fora do domicílio como a elevada densidade energética, o elevado teor de gorduras e açúcares, baixo conteúdo de fibras e cálcio, grandes porções de alimentos altamente palatáveis e ofertados em grande variedade e o consumo elevado de refrigerantes e doces contribuem para o ganho excessivo de peso. Conclusão: Constatada a importância da influência da alimentação fora do domicílio no ganho de peso, várias propostas são sugeridas para melhorar a oferta de alimentos saudáveis nas refeições realizadas fora de casa, no entanto, mais estudos são necessários para compreender quais mudanças no setor de alimentação fora de casa são efetivas para reduzir os impactos negativos na saúde da população.

Descritores: Alimentação Coletiva; Sobrepeso; Revisão.

\section{ABSTRACT}

Objective: To discuss the possible mechanisms of the relationship between eating away from home and excessive weight gain. Methods: A literature search was conducted to find scientific articles that described the characteristics of the foods consumed outside the home and articles that investigated the relationship between these characteristics and excessive weight gain published in the following databases: MEDLINE, Latin America and Caribbean Center on Health Sciences Information (LILACS) and Scientific Electronic Library Online (SciELO). Results: It was found that several characteristics of away-from-home foods such as high energy density, high fat and sugar content, poor fiber and calcium content, large portions of highly palatable foods available in great variety and high consumption of soft drinks and sweets contribute to excessive weight gain. Conclusion: After determining the importance of the influence of eating away from home on weight gain, several proposals are suggested to improve the availability of healthy foods in away-from-home meals; however, further studies are needed to understand which changes in the away-from-home food services are effective to reduce the negative impacts on the population's health. 


\section{RESUMEN}

Objetivo: Discutir los posibles mecanismos de la relación entre el consumo de alimentos fuera del domicilio y la ganancia excesiva de peso. Métodos: Se realizó una búsqueda bibliográfica de los artículos cientificos que describiesen las características de los alimentos consumidos fuera del domicilio y de artículos que investigasen la relación entre esas características y la ganancia excesiva de peso publicados en las bases de datos: MEDLINE, Biblioteca Latino-Americana e do Caribe (LILACS) e Scientific Eletronic Library Online (SciELO). Resultados: Se constató que las diversas características de la alimentación fuera del domicilio como la elevada densidad de energía, la elevada proporción de grasas y azucares, el bajo contenido de fibras y calcio, las grandes proporciones de alimentos muy agradables al paladar y ofrecidos en gran variedad y el consumo elevado de refrescos y dulces contribuyen para la ganancia excesiva de peso. Conclusión: Al constatar la importancia de la influencia de la alimentación fuera del domicilio para la ganancia de peso, varias propuestas son sugeridas para la mejoría de la oferta de alimentos saludables en las comidas realizadas fuera de casa. Sin embargo, más estudios son necesarios para la comprensión de cuales cambios en el sector de alimentación fuera de casa son efectivos para la reducción de los impactos negativos para la salud de la población.

Descriptores: Alimentación Colectiva; Sobrepeso; Revisión.

\section{INTRODUÇÃO}

As mudanças no estilo de vida da população ocasionaram modificações importantes nos hábitos alimentares das pessoas, como o aumento no consumo de alimentos prontos para consumo e na realização de refeições fora do lar. Em países desenvolvidos a alimentação realizada fora do domicílio contribui com uma parcela de até 39\% das calorias consumidas ${ }^{(1)}$. Nas áreas urbanas do Brasil, 18\% das calorias são provenientes de alimentos preparados e consumidos fora de casa ${ }^{(2)}$.

Os alimentos preparados e ou consumidos fora do domicílio vêm sendo apontados como tendo uma influência negativa na qualidade da $\operatorname{dieta}^{(1)}$. Um estudo proveniente do Inquérito de Saúde de São Paulo - ISA - conduzido em 2008 encontrou um índice de qualidade da refeição menor no almoço consumido fora do lar em comparação com o consumido dentro do $1 \operatorname{lar}^{(3)}$. Assim, o consumo de alimentos fora do domicílio é um dos fatores associados à dieta que parece ter uma contribuição considerável no aumento das prevalências de excesso de peso.

Essa possível relação entre comer fora de casa e ganho de peso tornou-se um problema de saúde pública e diversos estudos vêm sugerindo um impacto negativo desse hábito sobre o peso corporal ${ }^{(4,5)}$. Apesar de outros autores também apontarem possíveis explicações para a associação entre comer fora do lar e excesso de peso, como o tamanho das porções e a elevada densidade energética de alimentos preparados fora de casa, a discussão dos mecanismos que relacionam o consumo desses alimentos com o aumento do peso corporal ainda é insuficiente e pouco se sabe sobre a plausibilidade dessa relação.

O presente estudo tem como objetivo discutir os possíveis mecanismos da relação entre consumo de alimentos fora do domicílio e ganho excessivo de peso.

\section{MÉTODOS}

A pesquisa foi realizada utilizando as bases de dados MEDLINE, Biblioteca Latino-Americana e do Caribe (LILACS) e Scientific Eletronic Library Online (SciELO). Primeiramente investigaram-se as características dos alimentos consumidos/preparados fora do domicílio, utilizando-se as seguintes palavras-chave: away-from-home food, eating out, out-of-home eating, fast food, takeaway food, restaurant food combinadas com nutrient quality, diet quality, characteristics.

Após identificar as principais características dos alimentos consumidos/preparados fora de casa realizouse uma nova busca, investigando a relação entre as características identificadas e o ganho excessivo de peso. Utilizaram-se as seguintes palavras-chave: energy density, fat, saturated fat, fatty acids, sugar, soft drinks, sweetened beverages, sodium, iron, calcium, vitamin $D$, vitamin $C$, fiber, fruits, vegetables, whole cereals, food portion, variety e palatability combinadas com excessive weight, weight gain, obesity, overweight e body mass index. Foram incluídos artigos no idioma inglês e português publicados até março de 2016, sendo selecionados por meio de análise do título, resumo e texto completo, respectivamente.

\section{RESULTADOS}

Características relacionadas ao teor nutricional, a forma como os alimentos são oferecidos e o tipo de alimento oferecido fora do domicílio foram identificadas na revisão da literatura científica. A Figura 1 ilustra um esquema da possível relação entre essas características e o ganho de peso.

A realização de refeições fora do domicílio também foi associada a uma menor qualidade da dieta, incluindo baixo consumo de frutas, verduras e grãos integrais ${ }^{(1)}$. 


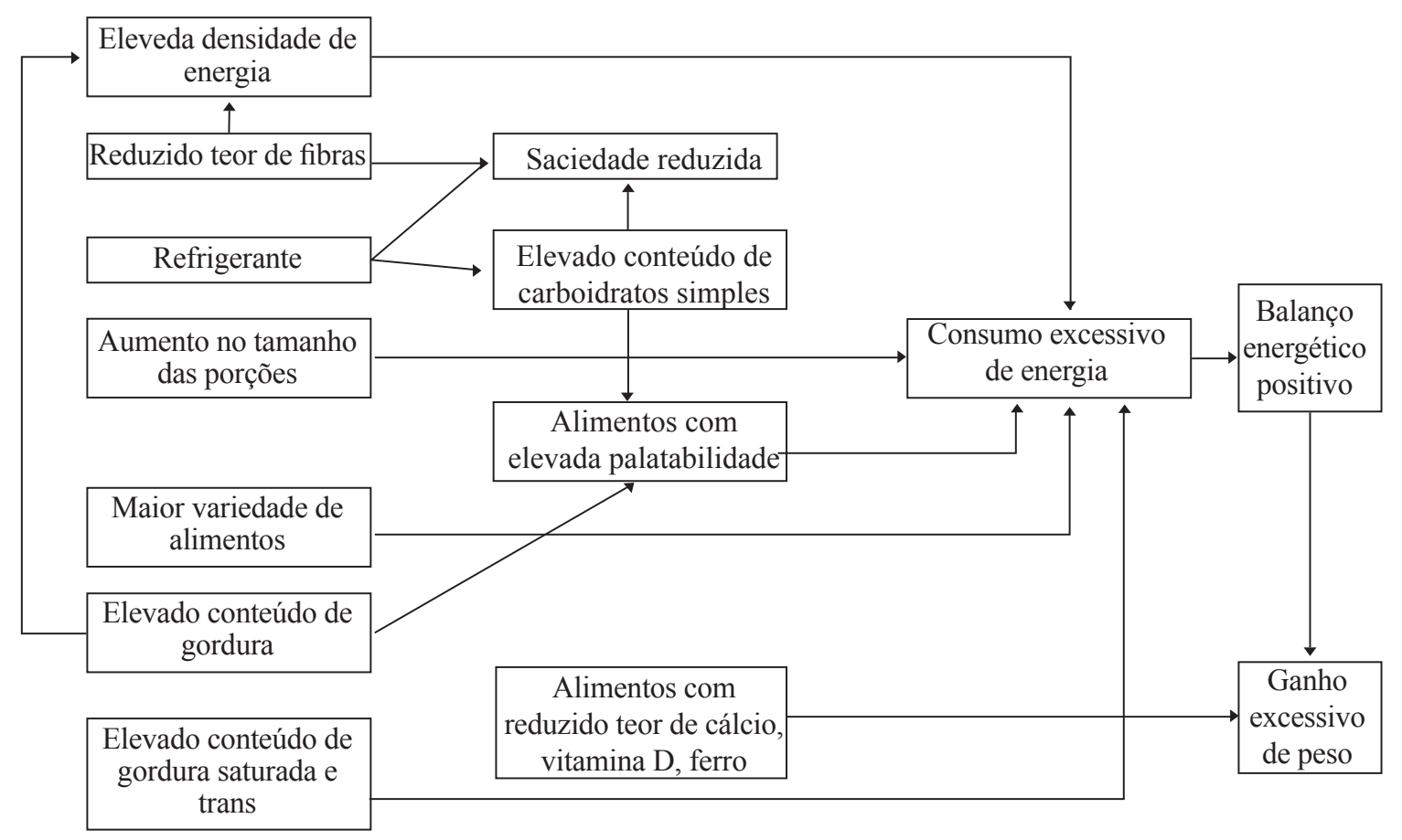

Figura 1 - Modelo da relação entre consumo de alimentos fora do domicílio e ganho de peso. Fortaleza, CE, 2016.

\section{DISCUSSÃO}

\section{Densidade energética e ganho de peso}

A relação entre densidade energética (razão entre a quantidade de energia e o peso do alimento pronto para o consumo) e o ganho de peso pode ser explicada pela capacidade limitada que os indivíduos têm para distinguir alimentos com conteúdo altamente energético e ajustar adequadamente a ingestão alimentar para manter a homeostase energética. Normalmente, tende-se a manter constante o volume dos alimentos consumidos, independente da variação na densidade de energia. Assim, o consumo de alimentos de alta densidade energética pode favorecer o aumento da ingestão passiva de energia e contribuir para o ganho de peso $^{(6)}$.

\section{Conteúdo de gordura, gordura saturada e gordura trans e ganho de peso}

O papel da gordura no ganho de peso é assunto de debate na literatura com evidências de que o consumo de dietas ricas em gordura favorece o ganho de peso, independente do consumo calórico total, e achados de que a gordura possui um efeito mínimo sobre a adiposidade.

Alguns mecanismos são propostos para explicar a relação entre ingestão de gordura e ganho de peso. O aumento no consumo de gordura não estimula proporcionalmente sua oxidação, sendo alta a eficiência com que os lipídeos da dieta são estocados como gordura corporal. Além disso, a termogênese induzida pela dieta após a ingestão de gordura é menor em comparação com a ingestão de carboidratos e proteínas. Ressalta-se também que as gorduras são absorvidas com maior eficiência no trato gastrointestinal quando comparadas com os carboidratos ${ }^{(7)}$.

Acredita-se ainda que dietas hiperlipídicas favoreçam hiperfagia devido à elevação da palatabilidade decorrente da presença de gordura $^{(8,9)}$. A relação entre consumo de gordura e ganho de peso também pode ser explicada por seu menor efeito na saciedade quando comparadas com os carboidratos ou proteínas, possivelmente devido à baixa habilidade de estimular a produção de insulina e leptina ${ }^{(9)}$.

Apesar de uma dieta pobre em gordura ser recomendada para a prevenção da obesidade, o efeito da redução de perda de peso decorrente da redução no consumo de gordura ainda é pequeno, sugerindo que o consumo calórico total é mais importante do que a composição da dieta para o peso corporal $^{(10)}$.

Quanto às gorduras saturadas, há evidências de que esse tipo de gordura possui um impacto diferenciado no balanço energético e consequentemente no peso corporal (11). Um estudo acompanhou 810 indivíduos acima de 25 
anos e encontraram que após 6 meses de intervenção, o consumo de gordura saturada foi positivamente associado ao peso corporal no $18^{\circ}$ mês do estudo ${ }^{(11)}$.

Alimentos preparados fora do domicílio também apresentam maior quantidade de gordura trans do que os preparados em casa, principalmente quando são alimentos fritos, como os servidos em restaurantes do tipo fast food ${ }^{(12)}$.

As gorduras trans vêm sendo fortemente associadas ao ganho de peso, à deposição de gordura abdominal e a doenças cardiovasculares, sendo verificadas fortes evidências acerca da associação entre consumo de gorduras trans e um aumento no peso corporal ${ }^{(13)}$.

\section{Consumo de bebidas açucaradas e alimentos ricos em açúcar e excesso de peso}

Os mecanismos de ganho de peso através do consumo de bebidas açucaradas ainda não estão totalmente elucidados, mas há indícios de que calorias provenientes de líquidos proporcionem menor saciedade em comparação com alimentos sólidos, resultando em maior consumo ${ }^{(14)}$.

A quantidade de açúcares refinados nessas bebidas confere uma alta carga glicêmica que, aliada a uma baixa saciedade, favorece um consumo de maiores quantidades, além de oportunizar o aumento da ingestão de outros alimentos. Um estudo recente ${ }^{(15)}$ encontrou que o aumento do peso corporal está relacionado ao consumo de bebidas açucaradas tanto em crianças como em adultos.

Por outro lado, a ausência do açúcar de adição nas bebidas, sendo substituído pelos edulcorantes artificias às bebidas de baixa caloria, também tem mostrado associação positiva com calorias extras de refeições sólidas, baixa ingestão de frutas e vegetais, e maior consumo de alimentos do tipo fast food e lanches hipercalóricos ${ }^{(16)}$.

\section{Conteúdo de fibras e peso corporal}

As fibras dietéticas têm diversas funções e seu efeito na alimentação está diretamente relacionado à sua atividade no trato gastrointestinal, afetando a fisiologia do apetite e a ingestão de energia. Sabe-se que os alimentos com maiores quantidades de fibras diminuem a ingestão de energia em razão das fibras não serem absorvidas no intestino. Logo, refeições ricas em fibras podem oferecer maior volume sem gerar um acréscimo de calorias ${ }^{(17)}$.

Além disso, os alimentos ricos em fibras têm menor valor calórico e menos palatabilidade quando comparados aos alimentos ricos em gordura ${ }^{(17)}$. Outro efeito das fibras dietéticas no controle da ingestão alimentar está relacionado às suas características físico-químicas. A capacidade de absorção de água e formação de géis no estômago causa um retardo no tempo de esvaziamento gástrico e sensação de plenitude que prolonga a saciedade ${ }^{(18)}$. O maior tempo de contato dos nutrientes na mucosa intestinal estimula a liberação de hormônios que regulam o apetite. Dessa forma, a ingestão de fibras pode prolongar a digestão e absorção dos carboidratos reduzindo a resposta da glicose pósprandial. A longo prazo isso pode melhorar a sensibilidade à insulina e estimular a lipólise, auxiliando na regulação do peso corporal ${ }^{(18)}$

Acredita-se que as fibras dietéticas também possam afetar a saciedade através das suas propriedades fermentativas. A ação de bactérias colônicas sobre as fibras produz ácidos graxos de cadeia curta. $\mathrm{O}$ incremento destes ácidos graxos poderia estimular as células do colón a produzirem hormônios, como o GLP-1 e o PYY, que aumentam a saciedade após uma refeição e diminuem a ingestão de alimentos, contribuindo para a manutenção da homeostase energética. Todavia, a relação causa e efeito ainda não está bem elucidada ${ }^{(17,18)}$.

\section{Micronutrientes e peso corporal}

Os alimentos preparados fora do domicílio têm sido caracterizados como alimentos de baixa qualidade nutricional, incluindo baixo teor de vitamina $\mathrm{C}$, cálcio $\mathrm{e}$ ferro e quantidade elevada de sódio(1).

A relação desses micronutrientes com o estado nutricional vem sendo amplamente estudada na literatura, já sendo descrito possível relação entre o consumo de micronutrientes e o excesso de peso. Além disso, verificouse que algumas vitaminas (A, C, E e D) e cálcio e zinco estão envolvidos em processos metabólicos e endócrinos da gênese e ou controle do excesso de peso, sendo importante a ingestão adequada desses nutrientes para se evitar o desenvolvimento da obesidade e de outras doenças crônicas não transmissíveis ${ }^{(19)}$.

Quanto ao cálcio, alguns estudos vêm demonstrando uma associação negativa desse mineral com o ganho excessivo de peso. Acredita-se que o cálcio se liga a ácidos graxos no trato gastrintestinal, impedindo sua absorção, além de aumentar a oxidação lipídica e favorecer a excreção de gordura $^{(20)}$.

Assim como o cálcio, a deficiência de vitamina D é um fator de risco independente para o desenvolvimento da gordura abdominal. As metaloproteinases de matriz (MMP) são enzimas proteolíticas essenciais no processo infeccioso e inflamatório, sendo responsáveis por remodelar a matriz extracelular e regular a migração dos leucócitos por meio da matriz. $\mathrm{O}$ tecido adiposo libera as MMP, confirmando o importante papel sobre a patogênese da obesidade ${ }^{(21)}$.

Em um estudo randomizado realizado com estudantes chineses com baixa ingestão de cálcio, a suplementação conjunta de cálcio e vitamina D3 resultou em perda 
significativa de gordura corporal e visceral ${ }^{(22)}$. A perda de tecido adiposo constatado no grupo usuário da formulação pode ser atribuída a alguns fatores dietéticos que tem como princípio a alta ingestão de cálcio, como a promoção da oxidação lipídica, a indução da apoptose adipocitária, a absorção lipídica reduzida a nível intestinal pela formação de sabões insolúveis excretados nas fezes, e a supressão da 1,25-dihidroxivitamina D (1,25-(OH)2D), responsável por diminuir o influxo intracelular de cálcio. Tais alterações intensificam a lipólise e reprimem a lipogênese nos adipócitos ${ }^{(20,22)}$.

Há evidências também que o calcitriol (vitamina D ativa) desempenhe função importante nos processos patológicos da obesidade por regular o nível de MMP e do inibidor tissular de metaloproteinase (TIMP) ${ }^{(22)}$.

A relação entre ferro e obesidade ainda não está muito bem estabelecida na literatura. Alguns autores sugerem uma relação inversa entre concentração de ferro e IMC, circunferência da cintura e massa gorda, descrevendo uma associação entre obesidade e baixa concentração de ferro ou deficiência de ferro ${ }^{(23)}$. Essa relação pode ser explicada pela baixa ingestão ou reduzida absorção do nutriente ou aumento da necessidade nutricional em indivíduos obesos devido ao sequestro de ferro decorrente da condição inflamatória. Uma revisão sobre o assunto descreveu que indivíduos obesos, apesar de apresentarem maior concentração de hemoglobina e ferritina, apresentam baixa saturação de transferrina, favorecendo o processo inflamatório associado à obesidade ${ }^{(24)}$. No entanto, os mecanismos causais dessa relação ainda não estão totalmente elucidados. Uma possível explicação seria o papel do hormônio hepcidina, que regula a homeostase do ferro, inibindo a absorção pelos enterócitos e favorecendo o sequestro do nutriente pelos macrófagos. A expressão do hormônio encontra-se elevada no estado inflamatório da obesidade devido à produção elevada de citocinas pró-inflamatória, como a interleucina 6 (IL-6) e o fator de necrose tumoral (TNF- $\alpha$ ), que estimulam a expressão da hepcidina ${ }^{(24)}$.

A vitamina $C$ é um potente antioxidante solúvel, envolvida também na síntese de colágeno e no sistema imunológico. Sua relação com a obesidade pode estar mediada por sua participação na síntese de catecolaminas, importante fonte regulatória da lipólise no tecido adiposo, e por sua atuação como cofator na síntese de carnitina, envolvida no transporte de ácidos graxos e na oxidação lipídica. Assim, a deficiência de vitamina $\mathrm{C}$ pode contribuir para a adipogênese $\mathrm{e}^{(19)}$.

Indivíduos obesos apresentam menores concentrações plasmáticas de vitamina $\mathrm{C}$ do que os que não são obesos. Estudo realizado com adultos e idosos de diferentes países europeus evidenciou que a baixa concentração plasmática de ácido ascórbico está relacionada à maior circunferência de cintura, independente do índice de massa corporal ${ }^{(25)}$.

\section{Tamanho das porções e consumo calórico}

O aumento no tamanho das porções dos alimentos consumidos fora do domicílio tem sido considerado um fator importante que contribui para o consumo elevado de calorias. Os mecanismos que favorecem o maior consumo de energia decorrente do aumento no tamanho das porções ainda não estão bem elucidados ${ }^{(26)}$. Sugerese que os indivíduos substituem ou ignoram os sinais de fome e saciedade quando consomem grandes porções e não há compensação nas refeições subsequentes dessa maior ingestão energética ${ }^{(27)}$.

A inconsistência no tamanho das porções (grandemédia-pequena) ofertadas também contribui com a maior ingestão de alimentos. Uma porção que antes era identificada como "grande" e agora é categorizada como "média" produz a falsa sensação de que a quantidade consumida é adequada. Um estudo ${ }^{(27)}$ que avaliou a percepção do tamanho das porções, o consumo e a percepção do mesmo verificou que os consumidores podem consumir grandes quantidades de alimentos que são ofertados como porção pequena e não perceberem que consumiram muito. Além disso, as pessoas mostraram-se menos culpadas ao ingerir porções menores, o que os autores denominaram de "gula sem culpa".

\section{Palatabilidade e variedade}

A palatabilidade dos alimentos decorre de características sensoriais, como sabor, cheiro, textura, temperatura e aparência visual, sendo a adição de açúcar, sal e gordura o que mais contribui para a palatabilidade dos alimentos. Além do sabor agradável, esses nutrientes ativam as vias de recompensa do cérebro que melhoram a sensibilidade e a palatabilidade ao alimento, estimulando o consumo exagerado. Acredita-se que no momento da degustação desses alimentos mais saborosos, a quantidade de dopamina cerebral se eleva, o que pode explicar, na maioria dos casos, a hiperfagia que leva à obesidade ${ }^{(28)}$.

Quanto à variedade dos alimentos, estudos têm mostrado que quanto maior a variedade de alimentos oferecidos em uma refeição maior o consumo energético. Indivíduos tendem a consumir mais alimentos quando maior variedade (número de alimentos com propriedades sensoriais diferentes) está disponível em uma mesma ocasião de consumo.

Isto ocorre provavelmente devido ao fato de que quanto mais alimentos de diferentes sabores, cores e formas de apresentação são oferecidos maior a ingestão individual de cada um deles até a completa apreciação. No entanto, um estudo destaca que o papel da variedade da dieta no controle 
do peso corporal não está bem definido e que o aumento da variedade em algumas categorias de alimentos (pobres em energia e ricos em nutrientes) e a redução da variedade em categorias com alimentos de alta densidade energética e baixa densidade de nutrientes, pode ser uma estratégia que promova aderência a padrões alimentares saudáveis e controle do peso corporal ${ }^{(29)}$.

Um estudo brasileiro mostrou que restaurantes por peso, que comercializam uma ampla variedade de opções de alimentos prontos para consumo, representam uma boa opção para quem opta por refeições mais saudáveis, indicando que é possível realizar refeições saudáveis fora de $\operatorname{casa}^{(30)}$.

\section{CONCLUSÃO}

A alimentação possui um papel importante tanto no desenvolvimento como na prevenção de doenças e mudanças nos hábitos alimentares, como a realização frequente de refeições fora de casa, afetam a qualidade da dieta e, consequentemente, a saúde pública de muitos países. Muitas evidências sugerem que as características da alimentação consumida fora do lar contribuem diretamente para o ganho excessivo de peso.

Essas características requerem atenção e merecem ser foco de futuros estudos, uma vez que a incorporação do hábito de se realizar refeições fora do lar é crescente no cotidiano da população e a influência dos alimentos preparados e ou consumidos fora de casa torna-se cada vez mais relevante na saúde dos consumidores. Apesar de ser possível alimentar-se de forma saudável fora do contexto domiciliar, é importante desenvolver estudos que apontem estratégias para melhorar a qualidade nutricional desses alimentos.

\section{REFERÊNCIAS}

1. Lachat C, Nago E, Verstraeten R, Roberfroid D, Van Camp J, Kolsteren P. Eating out of home and its association with dietary intake: a systematic review of the evidence. Obes Rev. 2012;13(4):329-46.

2. Bezerra IN, Moura Sousa A, Pereira RA, Sichieri R. Contribution of foods consumed away from home to energy intake in Brazilian urban areas: the 2008-9 Nationwide Dietary Survey. Br J Nutr. 2013;109(7):1276-83.

3. Gorgulho BM, Fisberg RM, Marchioni DM. Nutritional quality of major meals consumed away from home in Brazil and its association with the overall diet quality. Prev Med. 2013;57(2):98-101.
4. Bezerra IN, Curioni C, Sichieri R. Association between eating out of home and body weight. Nutr Rev. 2012;70(2):65-79.

5. Nago ES, Lachat CK, Dossa RA, Kolsteren PW. Association of out-of-home eating with anthropometric changes: a systematic review of prospective studies. Crit Rev Food Sci Nutr. 2014;54(9):1103-16.

6. Perez-Escamilla R, Obbagy JE, Altman JM, Essery EV, McGrane MM, Wong YP, et al. Dietary energy density and body weight in adults and children: a systematic review. J Acad Nutr Diet. 2012;112(5):671-84.

7. Jaworowska A, Blackham T, Davies IG, Stevenson L. Nutritional challenges and health implications of takeaway and fast food. Nutr Rev. 2013;71(5):310-8.

8. Prentice AM, Jebb SA. Fast foods, energy density and obesity: a possible mechanistic link. Obes Rev. 2003;4(4):187-94.

9. Pereira-Lancha LO, Coelho DF, Campos-Ferraz PL, Lancha AH Jr. Body fat regulation: is it a result of a simple energy balance or a high fat intake? J Am Coll Nutr. 2010;29(4):343-51.

10. Hooper L, Abdelhamid A, Bunn D, Brown $T$, Summerbell CD, Skeaff CM. Effects of total fat intake on body weight. Cochrane Database Syst Rev. 2015;8:CD011834.

11. Lin PH, Wang Y, Grambow SC, Goggins W, Almirall D. Dietary saturated fat intake is negatively associated with weight maintenance among the PREMIER participants. Obesity (Silver Spring). 2012;20(3): 571-5.

12. Stender S, Dyerberg J, Astrup A. Fast food: unfriendly and unhealthy. Int J Obes (Lond). 2007;31(6):887-90.

13. Thompson AK, Minihane AM, Williams CM. Trans fatty acids and weight gain. Int $\mathrm{J}$ Obes (Lond). 2011;35(3):315-24

14. Te Morenga L, Mallard S, Mann J. Dietary sugars and body weight: systematic review and meta-analyses of randomised controlled trials and cohort studies. BMJ. 2012;346:e7492.

15. Malik VS, Pan A, Willett WC, Hu FB. Sugar-sweetened beverages and weight gain in children and adults: a systematic review and meta-analysis. Am J Clin Nutr. 2013;98(4):1084-102.

16. Piernas C, Mendez MA, Ng SW, Gordon-Larsen P, Popkin BM. Low-calorie- and calorie-sweetened beverages: diet quality, food intake, and purchase 
patterns of US household consumers. Am J Clin Nutr. 2014;99(3):567-77.

17. Kristensen M, Jensen MG. Dietary fibres in the regulation of appetite and food intake. Importance of viscosity. Appetite. 2011;56(1):65-70.

18. Pereira MA, Ludwig DS. Dietary fiber and bodyweight regulation. Observations and mechanisms. Pediatrir Clin North Am. 2001;48(4):969-80.

19. Leao AL, Santos LC. Micronutrient consumption and overweight: is there a relationship?. Rev Bras Epidemiol. 2012;15(1):85-95.

20. Soares MJ, Murhadi LL, Kurpad AV, Chan She Ping-Delfos WL, Piers LS. Mechanistic roles for calcium and vitamin $\mathrm{D}$ in the regulation of body weight. Obes Rev. 2012;13(7):592-605.

21. Lương KVQ, Nguyễn LTH. The beneficial role of vitamin $\mathrm{D}$ in obesity: possible genetic and cell signaling mechanisms. Nutr J. 2013;12:89.

22. Zhu W, Cai D, Wang Y, Lin N, Hu Q, Qi Y, et al. Calcium plus vitamin D3 supplementation facilitated fat loss in overweight and obese college students with very-low calcium consumption: a randomized controlled trial. Nut J. 2013;12:8.

23. Chambers EC, Heshka S, Gallagher D, Wang J, PiSunyer FX, Pierson RN Jr. Serum iron and body fat distribution in a multiethnic cohort of adults living in New York City. J Am Diet Assoc. 2006;106(5):680-4.

24. Cheng HL, Bryant C, Cook R, O'Connor H, Rooney $\mathrm{K}$, Steinbeck K. The relationship between obesity and hypoferraemia in adults: a systematic review. Obes Rev. 2012;13(2):150-61.
25. Canoy D, Wareham N, Welch A, Bingham S, Luben $\mathrm{R}$, Day $\mathrm{N}$, et al. Plasma ascorbic acid concentrations and fat distribution in 19,068 British men and women in the European Prospective Investigation into Cancer and Nutrition Norfolk cohort study. Am J Clin Nutr. 2005;82(6):1203-9.

26. English L, Lasschuijt M, Keller KL.Mechanisms of the portion size effect. What is known and where do we go from here? Appetite. 2015;88:39-49.

27. Aydinoğlu NZ, Krishna A. Guiltless Gluttony: The Asymmetric Effect of Size Labels on Size Perceptions and Consumption. J Consum Res. 2011;37:105-1112.

28. Fortuna JL. The obesity epidemic and food addiction: clinical similarities to drug dependence. J Psychoactive Drugs. 2012;44(1):56-63.

29. Vadiveloo MK, Parekh N. Dietary variety: an overlooked strategy for obesity and chronic disease control.Am J Prev Med. 2015;49(6):974-9.

30. Santos MV, Proença RPC, Fiates GMR, Calvo MCM. Os restaurantes por peso no contexto de alimentação saudável fora de casa. Rev Nutr. 2011;24(4):641-9.

\section{Endereço para correspondência:}

Ilana Nogueira Bezerra

Universidade de Fortaleza - UNIFOR

Programa de Pós-Graduação em Saúde Coletiva - PPGSC

Avenida Washington Soares, 1321

Bairro: Edson Queiroz

CEP: 60811-905 - Fortaleza - CE - Brasil

E-mail: ilana.bezerra@yahoo.com.br 